\title{
VISCOUS DAMPING OF GRAVITY WAVES OVER A PERMEABLE BED
}

\author{
K. K. PURI \\ Department of Mathematics \\ University of Maine \\ Orono, Maine 04473 U.S.A.
}

(Received May 9, 1978 and in revised form June 19, 1978)

ABSTRACT. The damping of gravity waves over the surface of a layer of viscous fluid which overlies a porous bed saturated with the same fluid is studied. It is shown that viscosity may not be the dominant influence in the damping mechanism; the damping effects due to percolation in the fixed bed may be of the same or even higher order than those due to viscosity. KEY WORDS AND PHRASES. Waves in viscous liquids, gravity waves, permeable bed. AMS (MOS) SUBJECT CLASSIFICATION (1970) CODES.

1. INTRODUCTION.

In this paper we explore the role of permeability in the damping of viscous, gravity waves on the free surface of a layer of fluid. It is assumed that this layer is bounded below by a porous bed of infinite depth which is saturated with the same fluid.

Owing to the practical interest in such a study, this problem has already 
received attention from some authors. The main difficulty lies in the formulation of the appropriate boundary conditions at the interface. Putnam [1], followed by Reid and Kajura [2] considered the inviscid problem and calculated the effect of permeability of the porous medium on the damping of such waves. Their model was based upon the continuity of the pressure and the vertical velocity at the interface; the horizontal velocity thereat was left unaccounted. Hunt [3], generalized the above model by considering a viscous fluid, instead. In the way of interfacial conditions, he used the continuity of the vertical stress in place of that of the pressure and assumed zero horizontal velocity at the bed.

Two comments are germane to Hunt's conditions. Firstly, he included viscosity term in the expression for the stress in the bed. The same has also been assumed by Liu [6]. This is questionable because its effect is already included in Darcy drag term and so should not be considered again through the boundary conditions. The same has been recognized by Nield [4] and Murray [5]. In the present formulation, we equate the vertical stress in the fluid to the pressure alone in the bed. The second comment pertains to the assumption of zero horizontal fluid velocity at the bed surface. This would be valid for a rigid non-porous bottom but would not be consistent when motion of the fluid is permitted in the bed. To rectify the situation, Liu [6], instead, adopted the continuity of the horizontal component across the interface. This also is open to a similar objection. The validity of this condition would require that there should be two fluids, perhaps of different physical characteristics, but would not permit the presence of solid particles in the lower medium even if we assume a continuum flow which, indeed, we do. Murray, by requiring that the velocity components in the fluid match with the specific velocity components in the bed, adopts an intuitively more appealing condition. There, however, is no 
experimental evidence to support it. Also, he uses the seepage velocity in the linearized friction term. As a result the limiting case when the frequency becomes infinitely small cannot be reduced to Darcy's equation.

The question of relating horizontal velocity components across the interface has been a subject of several studies. The experimental studies of Beavers and Joseph [4], for example, lead to the conclusion that there is some net drag due to the transfer of forward momentum across the permeable interface, thus, negating one of the premises on which Hunt's study rests. They have also derived an empirical condition (see the equation (2.7), below), that describes the tangential flow in the vicinity of the boundary. Since then, their condition has found ample further support, both experimental and theoretical in the works of Saffman [8], Neale and Nadar [9], Beavers, Sparrow and Nasha [10], to mention a few. The same will be used in this work to study the effect of permeability and viscosity in the damping of two dimensional gravity waves.

\section{FORMULATION OF THE PROBLEM.}

We consider two-dimensional wave motion on the free surface of a layer of viscous, incompressible fluid of finite depth $\mathrm{h}$ and infinite horizontal extent, overlying an infinitely deep porous bed which is assumed to be saturated with the same fluid. The physical plane is taken to be the $\left(x^{\prime}, y^{\prime}\right)$ plane with $x^{\prime}$ axis along the free surface and $y^{\prime}$ axis vertically upward. Thus the bed surface is located at $y^{\prime}=-h$. Our problem is to find solutions which are small amplitude surface waves in the $\left(x^{\prime}, y^{\prime}\right)$ plane and to study the effect of viscosity and permeability on their decay.

The linearized Navier-Stokes equations of motion for the fluid and Darcy's equations for the bed are 


$$
\begin{gathered}
q_{1 t^{\prime}}^{\prime}=-\frac{1}{\rho} \nabla^{\prime} p_{1}^{\prime}+\nu \nabla^{\prime}{ }^{2} q_{I}^{\prime} \\
\frac{1}{n} q_{2 t^{\prime}}^{\prime}=-\frac{1}{\rho} \nabla^{\prime} p_{2}^{\prime}-D^{\prime}(n) q_{2}^{\prime}
\end{gathered}
$$

Here the subscripts ' 1 ' $\varepsilon$ '2' (used as ' $i$ ' below), refer to the fluid and the bed respectively. The quantities $\nabla^{\prime}=\left(\frac{\partial}{\partial x^{\prime}}, \frac{\partial}{\partial y},\right)$ and $q_{i}^{\prime} \equiv\left(u_{i}^{\prime}, \nu_{i}^{\prime}\right)$ represent the gradient and the velocities respectively. The latter satisfy the continuity equations,

$$
\nabla^{\prime} \cdot q_{1}^{\prime}=0 \text { and } \nabla^{\prime} \cdot q_{2}^{\prime}=0
$$

The gravitational effect is included in the pressure terms so as to write

$$
P_{i}^{\prime}=P_{i}^{\prime}-\rho g y-P_{0}
$$

where $\mathrm{P}_{i}$ are the hydrodynamical pressures in the two regimes, $\mathrm{P}_{0}$ is the constant. Also, $\rho$ is the viscosity of the fluid, $n$ is the porosity of the medium and $D^{\prime}(n)$ represents the drag in the fluid due to the solid particles in the porous matrix. We assume that the particles are fixed so that $D^{\prime}(n)$ may be taken as a constant, equivalent to $V / K^{\prime}$, where $K^{\prime}$ is the bed permeability.

The above equations are to be solved subject to the following boundary conditions. At the free surface we have:

$$
\begin{aligned}
& \text { kinematic condition: } v_{1}^{\prime}=n_{t^{\prime}}^{\prime} \text {, at } y^{\prime}=0 \\
& \text { vertical stress condition: }-P_{1}+2 v \rho v_{l y^{\prime}}^{\prime}=0 \text { at } y^{\prime}=0 \\
& \text { horizontal stress condition: } v\left(v_{l x^{\prime}}^{\prime}+u_{l y^{\prime}}^{\prime}\right)=0 \text { at } y^{\prime}=0
\end{aligned}
$$

At the interface we have,

$$
\begin{gathered}
v_{1}^{\prime}=v_{2}^{\prime} \text { at } y^{\prime}=-h \\
u_{i y^{\prime}}^{\prime}=\frac{\alpha}{\sqrt{K^{\prime}}}\left(u_{1}^{\prime}+\frac{1}{D}, p_{2 x^{\prime}}^{\prime}\right) \text { at } y^{\prime}=-h
\end{gathered}
$$

where $\alpha$ is a dimensionless number which depends upon the material of the porous matrix and is often called the slip constant. 


$$
-P_{1}^{\prime}+2 v \rho v_{l y^{\prime}}^{\prime}=-P_{2}^{\prime} \text { at } y^{\prime}=-h
$$

Finally, we require that

$$
\mathrm{q}_{2}^{\prime} \rightarrow 0 \text { as } \mathrm{y}^{\prime} \rightarrow-\infty
$$

The condition (2.7) is due to Beavers and Joseph. It finds its genesis in the observed fact that there is some net tangent drag due to the transfer of forward momentum at a permeable boundary. As a result of which the tangential component of the velocity of the fluid can be considerably greater than the mean filter velocity within the porous medium. Also, as discussed above, the viscosity term on the right of $(2.8)$ has been omitted on the ground that the drag due to the solid particles has already been accounted for in Darcy's equations. The present formulation differs from that of Hurt because of the difference between the equations $(2,7),(2.8)$ and their counterparts in his analysis. Assuming that $k$, w have the dimensions of the wave number and the frequency respectively, we define the dimensionless variables as follows:

$$
\begin{aligned}
& (x, y)=k\left(x^{\prime}, y^{\prime}\right), t=t^{\prime} w,\left(u_{j}, v_{j}\right)=\frac{k}{w}\left(u_{j}^{\prime}, v_{j}\right) \\
& P_{j}=\left(k^{2} / w^{2}\right) \rho^{-1} p_{j}^{\prime}, \quad F=\frac{9 k}{2} \quad \varepsilon=k^{2} v / w \\
& D=D^{\prime} / \rho w \quad \quad k=k^{2} K^{\prime} \quad n=n^{\prime} d
\end{aligned}
$$

We shall also write, $\lambda=\alpha / \sqrt{\mathrm{K}}, \overrightarrow{\mathrm{q}}_{j}=\nabla x \psi_{j}$.

Introducing the new variables in the above equations we get,

$$
\begin{gathered}
\left(\frac{\partial}{\partial t}-\varepsilon \nabla^{2}\right) \nabla^{2} \psi_{1}=0 \\
\left(\frac{\partial}{\partial t}+n D\right) \nabla^{2} \psi_{2}=0 \\
-\psi_{I x}=\eta_{t} \text { at } y=0 \\
P_{I}-F \eta+2 \varepsilon \psi_{I x y}=0 \text { at } y=0 \\
v\left(\psi_{I x x}-\psi_{I y y}\right)=0 \text { at } y=0
\end{gathered}
$$




$$
\begin{gathered}
\psi_{1 \mathrm{x}}=\psi_{2 \mathrm{x}} \text { at } \mathrm{y}=-\mathrm{kh} \\
\psi_{1 \mathrm{yy}}=\lambda\left(\psi_{1 \mathrm{y}}+\frac{1}{\mathrm{D}} \mathrm{P}_{2 \mathrm{x}}\right) \text { at } \mathrm{y}=-\mathrm{kh} \\
\mathrm{P}_{1}+2 \varepsilon \psi_{1 \mathrm{y} x}=\mathrm{P}_{2} \text { at } \mathrm{y}=-\mathrm{kh} \\
\psi_{2 \mathrm{x}}, \psi_{2 \mathrm{y}} \rightarrow 0 \text { as } \mathrm{y} \rightarrow-\infty
\end{gathered}
$$

Using the dimensionless form of the horizontal component of (2.1), we can easily eliminate $p_{1}$ from (2.14) to obtain,

$$
\varepsilon\left(3 \psi_{\text {lyxxt }}+\psi_{\text {lyyyt }}\right)-\psi_{\text {lytt }}-F_{x t}=0
$$

Using (2.13) to eliminate $n$ from (2.20), we get

$$
\varepsilon\left(3 \psi_{\text {lyxxt }}+\psi_{\text {lyyyt }}\right)-\psi_{\text {lytt }}+F \psi_{\text {lxx }}=0 \text { at } \mathrm{y}=0
$$

Similarly, using the horizontal components of the dimensionless forms of (2.1) and (2.2) to eliminate $p_{1}$ and $p_{2}$ from (2.18) and $p_{2}$ from (2.17), we get,

$$
\varepsilon\left(\psi_{\text {lyyy }}+3 \psi_{\text {lyxx }}\right)-\psi_{\text {lyt }}=-\left(\frac{1}{n} \psi_{2 y t}+D \psi_{2 y}\right) \text { at } y=-k h
$$

and

$$
\psi_{1 y y}=\lambda\left\{\psi_{1 y}-\psi_{2 y}-\frac{1}{n D} \psi_{2 y t}\right\} \quad \text { at } y=-k h
$$

3. SOLUTION OF THE PROBLEM.

We are interested in studying the progressive wave solutions of the form

$$
\psi_{j}=\psi_{j}(y) e^{i(t-x)}, j=1,2
$$

Substituting (3.1) in (2.11) \& (2.12) and invoking the condition (2.19) we get

$$
\begin{gathered}
\psi_{1}=A e^{y+k h}+B e^{(-y+h k)}+C e^{-\beta(y+k h)}+D e^{B y} \\
\psi_{2}=E e^{y+k h}
\end{gathered}
$$

where $B^{2}=1+i / \varepsilon$ and $A, B, C, D, E$ are constants of integration. The form of the solution (3.2) is motivated by the presence of the boundary layer at the free surface. Also using (3.1) in the boundary conditions (2.15), (2.22), 
(2.16), (2.22), (2.23) and discarding exponentially decaying terms, we have:

$$
\begin{gathered}
2 A \varepsilon e^{k h}+2 B \varepsilon e^{-k h}+D(2 \varepsilon+i)=0 \\
A e^{k h}(1-F-2 i \varepsilon)-B e^{-k h}(1+F-2 i \varepsilon)-D(F+2 i \varepsilon B)=0 \\
A+B+C-E=0 \\
A(2 \varepsilon+i)-B(2 \varepsilon+i)-2 \varepsilon B C-\left(\frac{i}{n}+D\right) E=0 \\
\varepsilon\left(\frac{1}{\lambda}-1\right) A+\varepsilon B\left(\frac{1}{\lambda}+1\right)+\varepsilon C\left(\frac{\beta^{2}}{\lambda}+B\right)+\varepsilon\left(1+\frac{i}{n D}\right) E=0
\end{gathered}
$$

We thus have five homogeneous equations in five unknowns, A, B, C, D, E, for a nontrivial solution to the above problem, we must have

$$
\left|\begin{array}{lllll}
2 \varepsilon e^{k h} & 2 \varepsilon e^{-k h} & 0 & 2 \varepsilon+i & 0 \\
e^{k h}(1-F-2 i \varepsilon) & -(1+F-2 i \varepsilon) e^{-k h} & 0 & -(F+2 i \varepsilon \beta) & 0 \\
1 & 1 & 1 & 0 & -1 \\
2 \varepsilon+i & -(2 \varepsilon+i) & -2 \varepsilon \beta & 0 & -D N \\
\varepsilon\left(\frac{1}{\lambda}-1\right) & \varepsilon\left(\frac{1}{\lambda}+1\right) & \varepsilon\left(\frac{\beta 2}{\lambda}+\beta\right) & 0 & \varepsilon N
\end{array}\right|=0
$$

where $N=1+i / n D$.

In order to find the unknowns $A, B, C$, etc., we use the third row in the determinant above, to obtain, after some simplification.

$$
\begin{gathered}
A \propto-\frac{1}{\lambda}\left(i+D N+i^{3 / 2} \varepsilon^{1 / 2}(D N+i)-\varepsilon\left(N+\frac{5}{\lambda}-\frac{2 D N i}{\lambda}+i N D\right)\right. \\
-i^{3 / 2} \varepsilon^{3 / 2}\left(\frac{5}{2} D N+2 N+\frac{2}{\lambda}-\frac{5}{2}\right)+o\left(\varepsilon^{2}\right) \\
B \propto \frac{1}{\lambda}(i-D N)+i^{3 / 2} \varepsilon^{1 / 2}(D N-i)+\varepsilon\left(\frac{5}{\lambda}+\frac{2 i N D}{\lambda}+i N D+N\right) \\
-i^{3 / 2} \varepsilon^{3 / 2}\left(\frac{5}{2} D N+\frac{2}{\lambda}+\frac{5}{2}+2 N\right)+0\left(\varepsilon^{2}\right) \\
C \propto 2 \varepsilon i(D N-i N-i / \lambda)+o\left(\varepsilon^{2}\right) \\
D \propto \frac{4 \varepsilon}{\lambda}(i D N \sinh k h-\cosh k h)+4 i^{3 / 2} \varepsilon^{3 / 2}(\cosh k h-i D N \sinh k h)+o\left(\varepsilon^{2}\right) \\
E \propto 4 \varepsilon(i \sinh k h+D N \cosh k h)\left(\frac{i}{\lambda}+\sqrt{i \varepsilon}\right)+o\left(\varepsilon^{2}\right)
\end{gathered}
$$


Also expanding the determinant in powers of $\varepsilon^{1 / 2}$ we obtain, after some tedious algebra,

$$
\begin{aligned}
F\left[\frac{1}{\lambda}(T\right. & \left.+\frac{i}{D N}\right)-(i \varepsilon)^{1 / 2}\left(i T-\frac{1}{D N}\right)+\varepsilon\left(i+\frac{1}{D}+\frac{3}{\lambda D N}\right) \\
& \left.+(i \varepsilon)^{3 / 2}\left(\frac{i T}{2}+\frac{2 T}{D}-\frac{1}{2 D N}+\frac{2 T}{\lambda D N}\right)\right] \\
& +\left[-\frac{1}{\lambda}\left(1+\frac{i T}{D N}\right)+(i \varepsilon)^{1 / 2}\left(i-\frac{T}{D N}\right)-\varepsilon\left(i T-\frac{4 i}{\lambda}+\frac{T}{D}+\frac{7 T}{\lambda D N}\right)\right. \\
& \left.+(i \varepsilon)^{3 / 2}\left(-\frac{9 i}{2}+\frac{4 i T}{\lambda}-\frac{2}{D}+\frac{9 T}{2 D N}-\frac{6}{\lambda D N}\right)\right]+o\left(\varepsilon^{2}\right)=0
\end{aligned}
$$

where $T=\tanh (k h)$.

The above equation, together with (2.10) yields the required dispersion relation for the wave frequency $w$ and, as a result, the wave form exp(iwt - ikx) as it evolves from an initially assumed form $\exp \left(i w_{0} t\right.$ - ikx), at any arbitrary time $t$, under the effects of viscosity of the fluid and the permeability characteristics of the porous bed.

In order to find the modes of vibrations, we set $\varepsilon=0$ in the equation (3.10). Realizing that $w=w_{0}$ in this case, we have, in terms of the dimensional variables,

$$
g k\left[i\left(1+\frac{T}{n}\right)+\frac{T D^{\prime}}{\rho w_{0}}\right]=w_{0}^{2}\left[i\left(T+\frac{1}{n}\right)+\frac{D^{\prime}}{\rho w_{0}}\right]
$$

Separating into the real and imaginary parts, we obtain,

$$
\mathrm{w}_{0}^{2}=\mathrm{gk} \tanh \mathrm{kh}
$$

and

$$
w_{0}^{2}=g k \frac{(\tanh k h+n)}{(1+n \tanh k h)} .
$$

These results agree with those of the afore mentioned authors.

We first deduce from the relation (3.10), the known viscous effect on the damping of a wave on the free surface of a fluid, bounded below by an impermeable, rigid bottom. This can be done by using two limiting processes. 
The first tends to replace the porous bed by an impermeable one and the second provides the vanishing of the horizontal slip at the bottom as a special case of the interfacial condition (2.24). They are accomplished by the limits, ND $\rightarrow \infty$, while holding $D$ fixed and $\lambda, D \rightarrow \infty$. Using these in the dispersion relation (3.10), we obtain

$$
w^{2}(T \sqrt{\varepsilon}-\sqrt{i})=g k(\sqrt{\varepsilon}-\sqrt{i T})
$$

where $\varepsilon=k^{2} \nu / w$.

$$
\begin{gathered}
\text { We now let } w=w_{0}+w_{1}, w_{1} \ll w_{0} \text { in equation (3.12) to obtain, } \\
w_{1}=-\frac{(1-i) k \sqrt{w_{0}}}{\sqrt{2} \sinh 2 k h}
\end{gathered}
$$

This gives both the frequency as well as the amplitude correction and agrees with the corresponding expressions in Weuhausen and Laitone [11].

\section{DISCUSSION OF THE RESULT.}

Strictly speaking, the role of permeability on the damping characteristics of surface waves depend, in a composite way, on various physical parameters of the problem. For a bed of course sand, the permeability $K^{\prime}=0\left(10^{-6}\right) \mathrm{cm}^{2}$. Assuming the kinematic viscosity of the water to be $0\left(10^{-2}\right) \mathrm{cm}^{2} / \mathrm{sec}$, we find $D^{\prime}=O\left(10^{4}\right)$. For water $\alpha=O(1)$ so that $\lambda=O\left(10^{3}\right)$. Under these conditions, it is clear from (3.10) that the permeability effect is negligible and the damping is dominated by viscosity and is of $0(\sqrt{\varepsilon})$. The presence of the porous bed is simply irrlelvant.

The above estimate of the magnitude of $K^{\prime}$ is correct only in deep waters. In shallow waters, particularly near the coast, the upward flow of water may increase the permeability and thus reduce the drag due to the bed. In this case $K^{\prime}$ may be $O\left(10^{-4}\right)$ or $O\left(10^{-3}\right)$ so that $D^{\prime}=O\left(10^{2}\right)$ or $D(10)$ and $\lambda=0\left(10^{-2}\right)$ or $0\left(10^{-1}\right)$ respectively. Guided by the procedure adopted in the derivation of the 
equation (3.14), we can obtain the frequency correction relevant to the new numerical data. Thus using the transformation (3.13) in the equation (3.10) and retaining only the dominating terms, we get, after some algebra,

$$
\operatorname{Im}\left(\mathrm{w}_{1}\right)=\frac{\mathrm{w}_{0}\left(1-\mathrm{T}^{2}\right)}{2 \mathrm{~T}}\left[\frac{\mathrm{D}_{0}+1 / \mathrm{n}}{\sqrt{2} \lambda \sqrt{\varepsilon_{0}}\left|\mathrm{M}_{0}\right|^{2}}+\frac{\mathrm{D}_{0}}{\left|\mathrm{M}_{0}\right| 2}+\frac{\sqrt{\varepsilon}}{\sqrt{2}}\right]
$$

where $M_{0}=N_{0} D_{0}$.

The quantities with the subscript zero, are the same as their unsubscripted analogues, scaled to the initial frequency $w_{0}$. Thus, for example,

$$
\varepsilon=\frac{\nu \mathrm{k}^{2}}{\mathrm{w}}, \varepsilon_{0}=\frac{\nu \mathrm{k}^{2}}{\mathrm{w}_{0}} \text {. }
$$

The equation (4.1) yields the frequency correction $\mathrm{w}_{1}$ to $\mathrm{w}_{0}$. To interpret it, we observe that $M_{0}=\frac{D^{\prime}}{w_{0}}+\frac{i}{n}$. This quantity in the present case could be $O(10)$ or $O(1)$ according as $w_{0}=O(1)$ or $O(10)$. In the first case, the bed porosity has contribution of the same order as viscosity and the two are additive. The second case entails the possibility of permeability being the dominating influence as an agent for the decay of surface waves. 


\section{REFERENCES}

1. Putnam, J. A. Loss of Wave Energy due to Percolation in a Permeable Sea Bottom, Trans. Am. Geophys. Union 30 (1949) 349-356.

2. Reid, R. O. and Kajiura, K. On the Damping of Gravity Waves Over a Permeable Sea Bed, Trans. Am. Geophys. Union 38 (1957) 662-666.

3. Hunt, J. M. On the Damping of Gravity Waves Propogated Over a Permeable Surface, J. Geophys. Res. 64 (1959) 437-442.

4. Nield, D. A. Onset of Convection in a Fluid Layer Overlying a Layer of a Porous Medium, J. Fluid Mech. 81 (1977) 517-522.

5. Murray, J. D. Viscous Damping of Gravity Waves Over a Permeable Bed, J. Geophys. Res. 70 (1965) 2325-2331.

6. Liu, Phillip Laf. Damping of Water Waves Over Porous Bed, J. Hyd. Div. HY 12 (1973) 2263-2271.

7. Beavers, G. S., Joseph, D. D. Boundary Conditions at a Naturally Permeable Wall, J. Fluid Mech. 30 (1967) 197-207.

8. Saffman, P. G. On the Boundary Conditions on the Surface of a Porous Medium, Studies Appl. Math. 50 (1971) 93-101.

9. Neale, G. and Nader, W. Practical Significance of Brinkman's Extension of Darcy's Law: Coupled Parallel Flows Within a Channel and a Bounding Porous Medium, Can. J. Chem. Engg. 52 (1974) 475-478.

10. Beavers, G. S. et al. Boundary Conditions at a Porous Surface Which Bounds a Fluid Flow, A. I. Ch. E. J. 20 (1974) 596-597.

11. Weuhausen, J. R. and Laitone, E. V. Hanbuch der Physik Band IX (1960).

12. Murray, J. D. On the Mathematics of Fluidization 1, Fundamental Equations and Wave Propogration, J. Fluid Mech. 2l (1965) 465-493. 


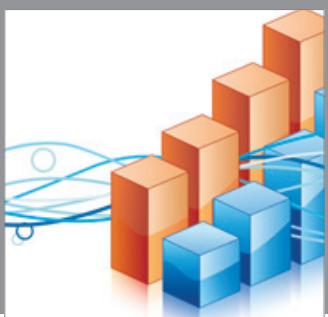

Advances in

Operations Research

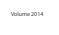

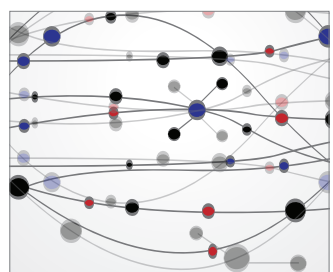

\section{The Scientific} World Journal
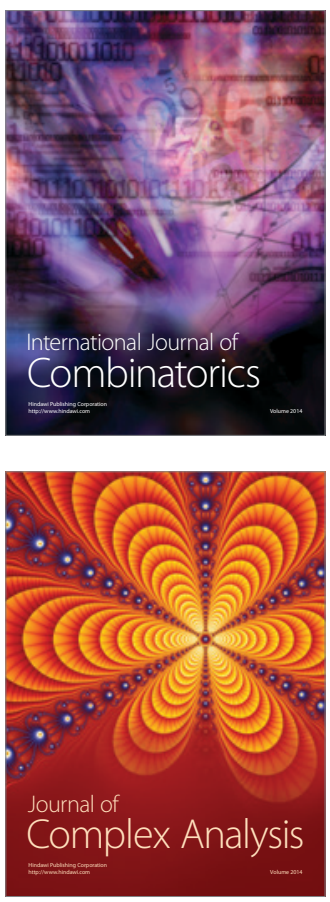

International Journal of

Mathematics and

Mathematical

Sciences
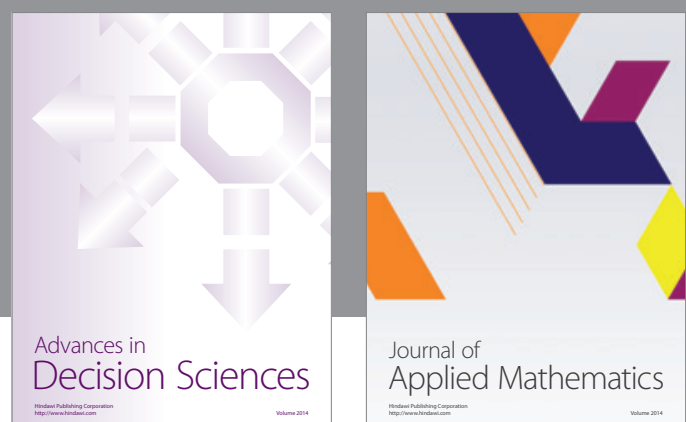

Journal of

Applied Mathematics
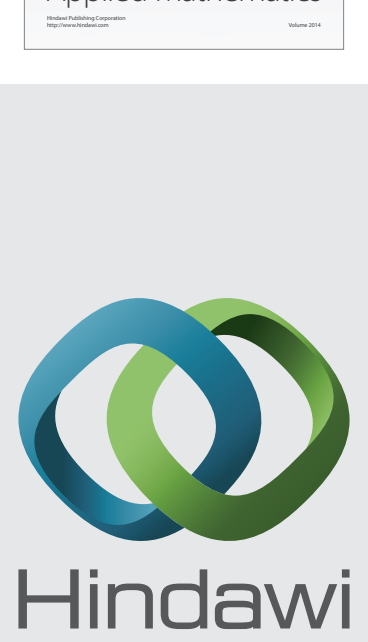

Submit your manuscripts at http://www.hindawi.com
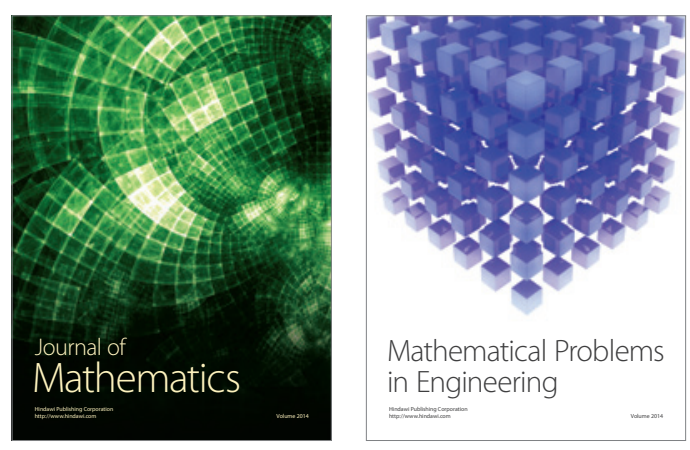

Mathematical Problems in Engineering
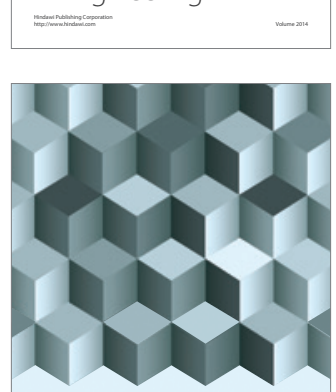

Journal of

Function Spaces
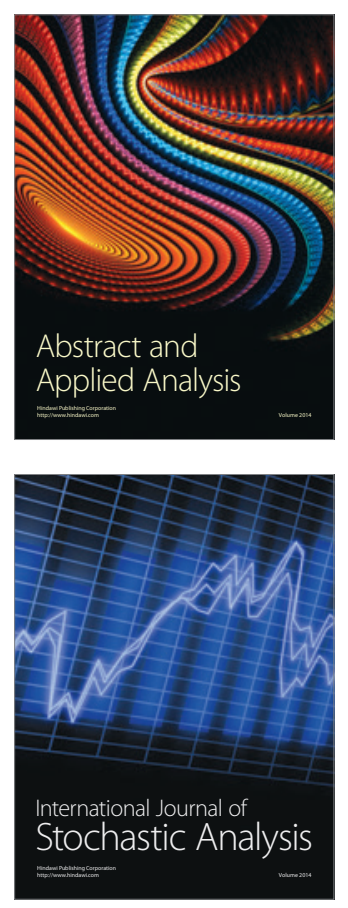

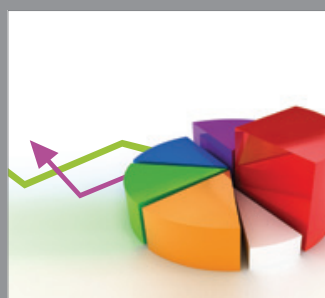

ournal of

Probability and Statistics

Promensencen
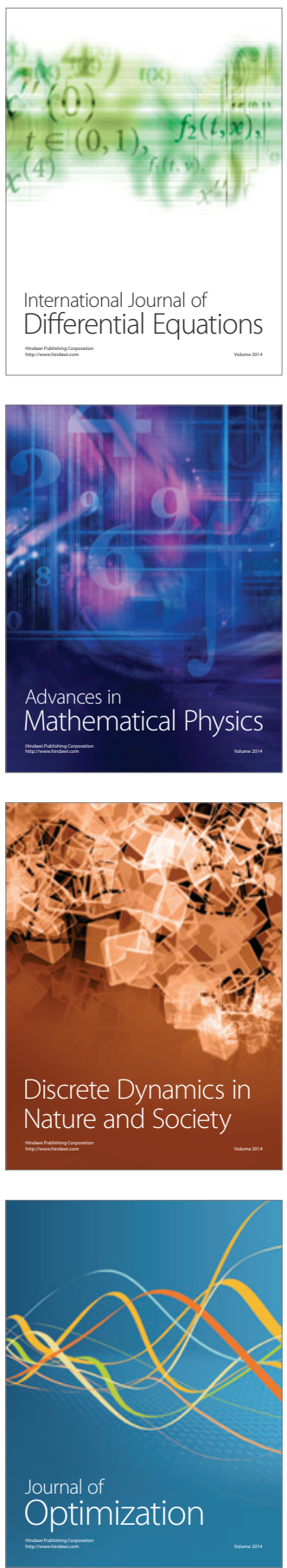